\title{
Initial bounds for analytic and bi-univalent functions by means of $(p, q)$-Chebyshev polynomials defined by differential operator
}

\author{
Ala Amourah ${ }^{\mathrm{a}, *}$ \\ ${ }^{a}$ Department of Mathematics, Faculty of Science and Technology, Irbid National University, Irbid, Jordan.
}

\begin{abstract}
In this paper, a subclass $\mathcal{T}_{\Sigma}^{\zeta}(m, \gamma, \lambda, p, q)$ of analytic and bi-univalent functions by means of $(p, q)$-Chebyshev polynomials is introduced. Certain coefficient bounds for functions belong to this subclass are obtained. In addition, the Fekete-Szegö problem is solved in this subclass.
\end{abstract}

Keywords: coefficient inequalities, bi-univalent functions, Fekete-Szegö problems.

2010 MSC: 30C45

\section{Introduction and preliminaries}

Let $\mathcal{A}$ denote the class of functions of the form:

$$
f(z)=z+\sum_{n=2}^{\infty} a_{n} z^{n}
$$

which are analytic in the open unit disk $\mathbb{U}=\{z:|z|<1\}$. Further, by $\mathcal{S}$ we shall denote the class of all functions in $\mathcal{A}$ which are univalent in $\mathbb{U}$. It is well known that every function $f \in \mathcal{S}$ has an inverse $f^{-1}$, defined by

$$
f^{-1}(f(z))=z \quad(z \in \mathbb{U})
$$

and

$$
f\left(f^{-1}(w)\right)=w \quad\left(|w|<r_{0}(f) ; r_{0}(f) \geq \frac{1}{4}\right)
$$

where

$$
f^{-1}(w)=w-a_{2} w^{2}+\left(2 a_{2}^{2}-a_{3}\right) w^{3}-\left(5 a_{2}^{3}-5 a_{2} a_{3}+a_{4}\right) w^{4}+\cdots .
$$

A function $f \in \mathcal{A}$ is said to be in $\Sigma$ the class of bi-univalent in $\mathbb{U}$ if both $f(z)$ and $f^{-1}(z)$ are univalent in $\mathbb{U}$. Lewin [9] showed that $\left|a_{2}\right|<1.51$ for every function $f \in \Sigma$ given by (1.1). Posteriorly, Brannan

\footnotetext{
*Corresponding author

Email address: alaammour@yahoo.com (Ala Amourah)
}

doi:10.31559/glm2019.7.2.1 
and Clunie [3] improved Lewin's result and conjectured that $\left|a_{2}\right| \leq \sqrt{2}$ for every function $f \in \Sigma$ given by (1.1). The coefficient estimate problem for each of the following Taylor Maclaurin coefficients:

$$
\left|a_{n}\right| \quad(n \in \mathbb{N} ; n \geq 4)
$$

It's still an open problem. Since then, there have been many researchers (see $[2,5,6,7,11,12,14,15,13]$ ) investigated several interesting subclasses of the class $\Sigma$ and found non-sharp estimates on the first two Taylor-Maclaurin coefficients $\left|a_{2}\right|$ and $\left|a_{3}\right|$. In fact, its worth to mention that by making use of the Faber polynomial coefficient expansions Jahangiri, Jay M., and Samaneh G. Hamidi [8] have obtained estimates for the general coefficients $\left|a_{n}\right|$ for bi-univalent functions subject to certain gap series.

For any integer $n \geq 2$ and $0<q<p \leq 1,(p, q)$-Chebyshev polynomials of the second kind is defined by the following recurrence relations:

$$
R_{n}(x, s, p, q)=\left(p^{n}+q^{n}\right) x R_{n-1}(x, s, p, q)+(p q)^{n-1} s R_{n-2}(x, s, p, q),
$$

with the initial values $R_{0}(x, s, p, q)=1$ and $R_{1}(x, s, p, q)=(p+q) x$ and $s$ is a variable.

Recently, Kızılateş, Naim and Bayram [16] defined $(p, q)-$ Chebyshev polynomials of the first and second kinds and derived explicit formulas, generating functions and some interesting properties of these polynomials.

The generating function of the $(p, q)$-Chebyshev polynomials of the second kind is as follows:

$$
H_{p, q}(z)=\frac{1}{1-x p z \tau_{p}-x q z \tau_{q}-s p q z^{2} \tau_{p, q}}=\sum_{n=0}^{\infty} R_{n}(x, s, p, q) z^{n} \quad(z \in \mathbb{U}) .
$$

where the Fibonacci operator $\tau_{q}$ Mason and Handscomb was introduced [17], by $\tau_{q} f(z)=f(q z)$,similarly, $\tau_{p, q} f(z)=f(p q z)$.

First off, we present some special cases of the polynomials $H_{p, q}(z)$ :

1. For $p=q=1$ and $s=-1$, we get the Chebyshev polynomials $R_{n}(x)$ of the second kind.

2. For $p=q=s=1$ and $x=\frac{x}{2}$, we get the Fibonacci polynomials $F_{n}(x)$.

3. For $p=q=1, s=2 y$ and $x=\frac{1}{2}$, we get the Jacobsthal polynomials $J_{n+1}(y)$.

4. If $p=q=s=1$, then we get the Pell polynomials $P_{n+1}(x)$.

Let $w(z)$ and $v(w)$ be two analytic functions in the unit disk $\mathbb{U}$ with $w(0)=v(0)=0,|w(z)|<$ $1,|v(z)|<1$, and suppose that

$$
w(z)=c_{1} z+c_{2} z^{2}+c_{3} z^{3}+\cdots, \text { and } v(w)=d_{1} w+d_{2} w^{2}+d_{3} w^{3}+\cdots \quad(z, w \in \mathbb{U}) .
$$

Making use of the binomial series

$$
(1-\gamma)^{m}=\sum_{j=0}^{m}\left(\begin{array}{c}
m \\
j
\end{array}\right)(-1)^{j} \gamma^{j} \quad\left(m \in \mathbb{N}, j \in \mathbb{N}_{0}=\mathbb{N} \cup\{0\}\right),
$$

recently for $f \in \mathcal{A}$, Frasin [4] defined the differential operator $A_{m, \lambda}^{\zeta} f(z)$ as follows:

$$
\begin{aligned}
A^{0} f(z) & =f(z), \\
A_{m, \gamma}^{1} f(z) & =(1-\gamma)^{m} f(z)+\left(1-(1-\gamma)^{m}\right) z f^{\prime}(z)=A_{m, \lambda} f(z), \gamma>0 ; m \in \mathbb{N}, \\
A_{m, \gamma}^{\zeta} f(z) & =A_{m, \gamma}\left(A^{\zeta-1} f(z)\right) \\
& =z+\sum_{n=2}^{\infty}\left[1+(n-1) C_{j}^{m}(\gamma)\right]^{\zeta} a_{n} z^{n} ; \zeta \in \mathbb{N},
\end{aligned}
$$


where

$$
C_{j}^{m}(\gamma)=\sum_{j=1}^{m}\left(\begin{array}{c}
m \\
j
\end{array}\right)(-1)^{j+1} \gamma^{j}
$$

Using the relation (1.4), it is easily verified that

$$
C_{j}^{m}(\gamma) z\left(A_{m, \gamma}^{\zeta} f(z)\right)^{\prime}=A_{m, \gamma}^{\zeta+1} f(z)-\left(1-C_{j}^{m}(\gamma)\right) A_{m, \gamma}^{\zeta} f(z)
$$

By specializing the parameters we observe that, for $m=1, A_{1, \lambda}^{\zeta}$ defined by Al-Oboudi [1] and for $m=\gamma=1, A_{1,1}^{\zeta}$ defined by Sălăgean [10].

\section{The function class $\mathcal{T}_{\Sigma}^{\zeta}(m, \gamma, \lambda, p, q)$}

Definition 2.1. A function $f(z) \in \Sigma$ is said to be in the class $\mathcal{T}_{\Sigma}^{\zeta}(m, \gamma, \lambda, p, q)$ if and only if

$$
(1-\lambda) \frac{A_{m, \gamma}^{\zeta} f(z)}{z}+\lambda\left(A_{m, \gamma}^{\zeta} f(z)\right)^{\prime} \prec H_{p, q}(z)=\frac{1}{1-x p z \tau_{p}-x q z \tau_{q}-s p q z^{2} \tau_{p, q}}
$$

and

$$
(1-\lambda) \frac{A_{m, \gamma}^{\zeta} g(w)}{w}+\lambda\left(A_{m, \gamma}^{\zeta} g(w)\right)^{\prime} \prec H_{p, q}(w)=\frac{1}{1-x p w \tau_{p}-x q w \tau_{q}-s p q w^{2} \tau_{p, q}}
$$

where $0 \leq \lambda \leq 1 z$, w in $\mathbb{U}$ and $g(w)=f^{-1}(w)$.

\section{Coefficient bounds for the function class $\mathcal{T}_{\Sigma}^{\zeta}(m, \gamma, \lambda, p, q)$}

We begin with the following result involving initial coefficient bounds for the function class $\mathcal{T}_{\Sigma}^{\zeta}(m, \gamma, \lambda, p, q)$.

Theorem 3.1. If $f(z)$ given by (1.1) is in the class $\mathcal{T}_{\Sigma}^{\zeta}(m, \gamma, \lambda, p, q)$. Then

$$
\left|a_{2}\right| \leq \frac{(p+q) x \sqrt{(p+q) x}}{\sqrt{\chi}}
$$

and

$$
\left|a_{3}\right| \leq \frac{(p+q)^{2} x^{2}}{\chi}+\frac{(p+q) x}{(1+2 \lambda)\left(1+2 C_{j}^{m}(\gamma)\right)^{\zeta}}
$$

where

$$
\chi=\left|\left(1+2 C_{j}^{m}(\gamma)\right)^{\zeta}(1+2 \lambda)(p+q)^{2} x^{2}-\left[\left(p^{2}+q^{2}\right)(p+q) x^{2}+p q s\right]\left(\left(1+C_{j}^{m}(\gamma)\right)^{\zeta}(1+\lambda)\right)^{2}\right| .
$$

Proof. Let $f(z) \in \mathcal{T}_{\Sigma}^{\zeta}(m, \gamma, \lambda, p, q)$. Then there are analytic functions $u$ and $v$, with $u(0)=v(0)=0$, $|u(z)|<1,|v(z)|<1$, given by (1.3) and satisfying the following conditions:

$$
(1-\lambda) \frac{A_{m, \gamma}^{\zeta} f(z)}{z}+\lambda\left(A_{m, \gamma}^{\zeta} f(z)\right)^{\prime}=H_{p, q}(w(z))
$$

and

$$
(1-\lambda) \frac{A_{m, \gamma}^{\zeta} g(w)}{w}+\lambda\left(A_{m, \gamma}^{\zeta} g(w)\right)^{\prime}=H_{p, q}(v(w))
$$

where $g(w)=f^{-1}(w)$. 
or some analytic functions

$$
w(z)=c_{1} z+c_{2} z^{2}+c_{3} z^{3}+\cdots \quad(z \in \mathbb{U}),
$$

and

$$
v(w)=d_{1} w+d_{2} w^{2}+d_{3} w^{3}+\cdots \quad(w \in \mathbb{U}),
$$

such that $w(0)=v(0)=0,|w(z)|<1(z \in \mathbb{U})$ and $|v(w)|<1 \quad(w \in \mathbb{U})$.

It follows from (3.3) and (3.4) that

$$
\begin{aligned}
& (1-\lambda) \frac{A_{m, \gamma}^{\zeta} f(z)}{z}+\lambda\left(A_{m, \gamma}^{\zeta} f(z)\right)^{\prime} \\
& =1+R_{1}(x, s, p, q) c_{1} z+\left[R_{1}(x, s, p, q) c_{2}+R_{2}(x, s, p, q) c_{1}^{2}\right] z^{2}+\cdots
\end{aligned}
$$

and

$$
\begin{aligned}
& (1-\lambda) \frac{A_{m, \gamma}^{\zeta} g(w)}{w}+\lambda\left(A_{m, \gamma}^{\zeta} g(w)\right)^{\prime} \\
& \left.=1+R_{1}(x, s, p, q) d_{1} w+\left[R_{1}(x, s, p, q) d_{2}+R_{2}(x, s, p, q) d_{1}^{2}\right]\right) w^{2}+\cdots
\end{aligned}
$$

A short calculation shows that

$$
\begin{aligned}
(1+\lambda)\left(1+C_{j}^{m}(\gamma)\right)^{\zeta} a_{2} & =R_{1}(x, s, p, q) c_{1}, \\
(1+2 \lambda)\left(1+2 C_{j}^{m}(\gamma)\right)^{\zeta} a_{3} & =R_{1}(x, s, p, q) c_{2}+R_{2}(x, s, p, q) c_{1}^{2}, \\
-(1+\lambda)\left(1+C_{j}^{m}(\gamma)\right)^{\zeta} a_{2} & =R_{1}(x, s, p, q) d_{1},
\end{aligned}
$$

and

$$
(1+2 \lambda)\left(1+2 C_{j}^{m}(\gamma)\right)^{\zeta}\left(2 a_{2}^{2}-a_{3}\right)=R_{1}(x, s, p, q) d_{2}+R_{2}(x, s, p, q) d_{1}^{2} .
$$

From (3.7) and (3.9), we get

$$
c_{1}=-d_{1}
$$

and

$$
2\left[\left(1+C_{j}^{m}(\gamma)\right)^{\zeta}(1+\lambda)\right]^{2} a_{2}^{2}=R_{1}^{2}(x, s, p, q)\left(c_{1}^{2}+d_{1}^{2}\right) .
$$

By adding (3.8) to (3.10), we have

$$
2\left(1+2 C_{j}^{m}(\gamma)\right)^{\zeta}(1+2 \lambda) a_{2}^{2}=R_{1}(x, s, p, q)\left(c_{2}+d_{2}\right)+R_{2}(x, s, p, q)\left(c_{1}^{2}+d_{1}^{2}\right) .
$$

Therefore, from equalities (3.12) and (3.13) we find that

$$
\begin{aligned}
& {\left[2\left(1+2 C_{j}^{m}(\gamma)\right)^{\zeta}(1+2 \lambda) R_{1}^{2}(x, s, p, q)-2 R_{2}(x, s, p, q)\left(\left(1+C_{j}^{m}(\gamma)\right)^{\zeta}(1+\lambda)\right)^{2}\right] a_{2}^{2} } \\
= & R_{1}^{3}(x, s, p, q)\left(c_{2}+d_{2}\right) .
\end{aligned}
$$

Then

$$
\left|a_{2}\right| \leq \frac{(p+q) x \sqrt{(p+q) x}}{\sqrt{\chi}}
$$

where

$$
\chi=\left|\left(1+2 C_{j}^{m}(\gamma)\right)^{\zeta}(1+2 \lambda)(p+q)^{2} x^{2}-\left[\left(p^{2}+q^{2}\right)(p+q) x^{2}+p q s\right]\left(\left(1+C_{j}^{m}(\gamma)\right)^{\zeta}(1+\lambda)\right)^{2}\right| .
$$

Next, in order to find the bound on $\left|a_{3}\right|$, subtracting (3.10) from (3.8) and using (3.11), we get

$$
2(1+2 \lambda)\left(1+2 C_{j}^{m}(\gamma)\right)^{\zeta} a_{3}=2(1+2 \lambda)\left(1+2 C_{j}^{m}(\gamma)\right)^{\zeta} a_{2}^{2}+R_{1}(x, s, p, q)\left(c_{2}-d_{2}\right) .
$$


Then in view of (3.15) and (3.11), we have

$$
2(1+2 \lambda)\left(1+2 C_{j}^{m}(\gamma)\right)^{\zeta}\left|a_{3}\right| \leq 2(1+2 \lambda)\left(1+2 C_{j}^{m}(\gamma)\right)^{\zeta}\left|a_{2}\right|^{2}+2 R_{1}(x, s, p, q)
$$

From (3.7), we immediately have

$$
\left|a_{3}\right| \leq\left|a_{2}\right|^{2}+\frac{(p+q) x}{(1+2 \lambda)\left(1+2 C_{j}^{m}(\gamma)\right)^{\zeta}}
$$

Now the assertion (3.2) follows from (3.1). This evidently completes the proof of Theorem 4.1.

By taking $\lambda=1$ in Theorem 3.1, we have

Corollary 3.2. If $f(z)$ given by (1.1) is in the class $\mathcal{T}_{\Sigma}^{\zeta}(m, \gamma, p, q)$. Then

$$
\left|a_{2}\right| \leq \frac{(p+q) x \sqrt{(p+q) x}}{\sqrt{\left|3\left(1+2 C_{j}^{m}(\gamma)\right)^{\zeta}(p+q)^{2} x^{2}-4\left[\left(p^{2}+q^{2}\right)(p+q) x^{2}+p q s\right]\left(\left(1+C_{j}^{m}(\gamma)\right)^{\zeta}\right)^{2}\right|}}
$$

and

$$
\left|a_{3}\right| \leq \frac{(p+q)^{2} x^{2}}{\tau}+\frac{(p+q) x}{3\left(1+2 C_{j}^{m}(\gamma)\right)^{\zeta}}
$$

where

$$
\tau=\left|3\left(1+2 C_{j}^{m}(\gamma)\right)^{\zeta}(p+q)^{2} x^{2}-4\left[\left(p^{2}+q^{2}\right)(p+q) x^{2}+p q s\right]\left(\left(1+C_{j}^{m}(\gamma)\right)^{\zeta}\right)^{2}\right|
$$

\section{Fekete-Szegö inequalities for the function class $\mathcal{T}_{\Sigma}^{\zeta}(m, \gamma, \lambda, p, q)$}

Now, we are ready to find the sharp bounds of Fekete-Szegö functional $a_{3}-\delta a_{2}^{2}$ defined for $f \in$ $\mathcal{T}_{\Sigma}^{\zeta}(m, \gamma, \lambda, p, q)$ given by (1.1).

Theorem 4.1. Let $f(z)$ given by (1.1), be in the class $\mathcal{T}_{\Sigma}^{\zeta}(m, \gamma, \lambda, p, q)$. Then

$$
\left|a_{3}-\delta a_{2}^{2}\right| \leq\left\{\begin{array}{c}
\frac{(p+q) x}{(1+2 \lambda)\left(1+2 C_{j}^{m}(\gamma)\right)^{\zeta}} \quad \text { for } 0 \leq|h(\delta)|<\frac{1}{2(1+2 \lambda)\left(1+2 C_{j}^{m}(\gamma)\right)^{\zeta}} \\
2(p+q)|h(\delta)| x \quad \text { for } \quad|h(\delta)| \geq \frac{1}{2(1+2 \lambda)\left(1+2 C_{j}^{m}(\gamma)\right)^{\zeta}}
\end{array},\right.
$$

where

$$
h(\delta)=\frac{(p+q)^{2} x^{2}(1-\delta)}{2\left[\left(1+2 C_{j}^{m}(\gamma)\right)^{\zeta}(1+2 \lambda)(p+q)^{2} x^{2}-\left[\left(p^{2}+q^{2}\right)(p+q) x^{2}+p q s\right]\left(\left(1+C_{j}^{m}(\gamma)\right)^{\zeta}(1+\lambda)\right)^{2}\right]} .
$$

Proof. From (3.14) and (3.15), we get

$$
a_{2}^{2}=\frac{R_{1}^{3}(x, s, p, q)\left(c_{2}+d_{2}\right)}{2\left[\left(1+2 C_{j}^{m}(\gamma)\right)^{\zeta}(1+2 \lambda) R_{1}^{2}(x, s, p, q)-R_{2}(x, s, p, q)\left(\left(1+C_{j}^{m}(\gamma)\right) \zeta(1+\lambda)\right)^{2}\right]}
$$

and 


$$
a_{3}=\frac{2(1+2 \lambda)\left(1+2 C_{j}^{m}(\gamma)\right)^{\zeta} a_{2}^{2}+R_{1}(x, s, p, q)\left(c_{2}-d_{2}\right)}{2(1+2 \lambda)\left(1+2 C_{j}^{m}(\gamma)\right)^{\zeta}}
$$

From the equations (4.2) and (4.3), it follows that

$$
\begin{aligned}
a_{3}-\delta a_{2}^{2}= & R_{1}(x, s, p, q)\left[\left(h(\delta)+\frac{1}{2(1+2 \lambda)\left(1+2 C_{j}^{m}(\gamma)\right)^{\zeta}}\right) c_{2}\right. \\
& \left.+\left(h(\delta)-\frac{1}{2(1+2 \lambda)\left(1+2 C_{j}^{m}(\gamma)\right)^{\zeta}}\right) d_{2}\right]
\end{aligned}
$$

where

$$
h(\delta)=\frac{R_{1}^{2}(x, s, p, q)(1-\delta)}{2\left[\left(1+2 C_{j}^{m}(\gamma)\right)^{\zeta}(1+2 \lambda) R_{1}^{2}(x, s, p, q)-R_{2}(x, s, p, q)\left(\left(1+C_{j}^{m}(\gamma)\right) \zeta(1+\lambda)\right)^{2}\right]} .
$$

Then, we easily conclude that

$$
\left|a_{3}-\delta a_{2}^{2}\right| \leq \begin{cases}\frac{(p+q) x}{(1+2 \lambda)\left(1+2 C_{j}^{m}(\gamma)\right)^{\zeta}}, & |h(\delta)| \leq \frac{1}{2(1+2 \lambda+6 \mu)} \\ 2(p+q)|h(\delta)| x, & |h(\delta)| \geq \frac{1}{2(1+2 \lambda+6 \mu)}\end{cases}
$$

This proves Theorem 4.1.

By taking $\lambda=1$ in Theorem 4.1, we have

Corollary 4.2. Let $f(z)$ given by (1.1), be in the class $\mathcal{T}_{\Sigma}^{\zeta}(m, \gamma, p, q)$. Then

$$
\left|a_{3}-\delta a_{2}^{2}\right| \leq\left\{\begin{array}{l}
\frac{(p+q) x}{3\left(1+2 C_{j}^{m}(\gamma)\right)^{\zeta}} \quad \text { for } 0 \leq|h(\delta)|<\frac{1}{6\left(1+2 C_{j}^{m}(\gamma)\right)^{5}} \\
2(p+q)|h(\delta)| x \quad \text { for } \quad|h(\delta)| \geq \frac{1}{6\left(1+2 C_{j}^{m}(\gamma)\right)^{\zeta}}
\end{array}\right.
$$

where

$$
h(\delta)=\frac{(p+q)^{2} x^{2}(1-\delta)}{2\left[3\left(1+2 C_{j}^{m}(\gamma)\right)^{\zeta}(p+q)^{2} x^{2}-4\left[\left(p^{2}+q^{2}\right)(p+q) x^{2}+p q s\right]\left(\left(1+C_{j}^{m}(\gamma)\right)^{\zeta}\right)^{2}\right]}
$$

\section{References}

[1] T. Al-Hawary, A. A. Amourah, M. Darus, Differential sandwich theorems for $p$-valent functions associated with two generalized differential operator and integral operator, International Information Institute (Tokyo). Information 17 (8) (2014), 3559.1

[2] A. A. Amourah and F. Yousef, Some properties of a class of analytic functions involving a new generalized differential operator, Boletim da Sociedade Paranaense de Matemática 38(6) (2018), 33-42. https://doi.org/10.5269/bspm.v38i6.40530 1

[3] A. Amourah, Faber polynomial coefficient estimates for a class of analytic bi-univalent functions, AIP Conference Proceedings. 2096(1)(2019). 1

[4] A. Amourah and M. Darus, Some properties of a new class of univalent functions involving a new generalized differential operator with negative coefficients, Indian Journal of Science and Technology,9 (2016), 1-7. https: / / doi.org/10.17485/ijst/2016/v9i36/97738 1

[5] S. Altınkaya, and S. Yalçın, The $(p, q)$-Chebyshev polynomial bounds of a general bi-univalent function class, Boletín de la Sociedad Matemática Mexicana (2019), 1-8. https:/ / doi.org/10.1007/s40590-019-00246-2 1

[6] F. M. Al-Oboudi, On univalent functions defined by generalized Salagean operator, Int. J. Math. Sci., (2004), $1429-1436$. https: / / doi.org/10.1155/s0161171204108090 1

[7] D. A. Brannan, J.G. Clunie (Eds.), Aspects of Contemporary Complex Analysis (Proceedings of the NATO Advanced Study Institute held at the University of Durham, Durham; July 1 20, 1979), Academic Press, New York and London, (1980). 1 
[8] S. Bulut, Coefficient estimates for a class of analytic and bi-univalent functions, Novi Sad J. Math., 43( 2)(2013), 59-65. 1

[9] B. A. Frasin, A new differential operator of analytic functions involving binomial series, Boletim da Sociedade Paranaense de Matemática 38.5 (2020), 205-213. https:// doi.org/10.5269/bspm.v38i5.40188 1

[10] C. Kızılate, N. Tuğlu, B. Çekim, On the $(p, q)$-Chebyshev polynomials and related polynomials, Mathematics, 7 (2019), 1-12. https://doi.org/10.3390/math7020136 1

[11] J. C. Mason, D. C. Handscomb, Chebyshev Polynomials, Chapman \& Hall, Boca Raton (2003). 1

[12] J. M. Jahangiri and S. G. Hamidi, Coefficient estimates for certain classes of bi-univalent functions, Int. J. Math and Math. Sci. (2013), Article ID 190560. https:/ / doi.org/10.1155/2013/190560 1

[13] G. S. Sălăgean, Subclasses of univalent functions, Lecture Notes in Math., 1013 (1983), 362-372, Springer-Verlag, Heidelberg. 1

[14] H. M. Srivatava and D. Bansal, Coefficient estimates for a subclass of analytic and bi-univalent functions, J. Egyptian Math. Soc. 23 (2015), 242-246. 1

[15] F. Yousef, T. Al-Hawary and G. Murugusundaramoorthy, Fekete-Szegö functional problems for some subclasses of bi-univalent functions defined by Frasin differential operator. Afrika Matematika 30.3-4 (2019), 495-503. https:/ / doi.org/10.1007/s13370-01900662-7 1

[16] F. Yousef, B. A. Frasin and T. Al-Hawary, Fekete-Szegö Inequality for Analytic and Bi-univalent Functions Subordinate to Chebyshev Polynomials, arXiv preprint arXiv:1801.09531 (2018). 1 